\title{
Developmental changes in barley microRNA expression profiles coupled with miRNA target analysis
}

\author{
Andrzej Pacak ${ }^{* \otimes}$, Katarzyna Kruszka ${ }^{*}$, Aleksandra Swida-Barteczka1*, Przemyslaw Nuc ${ }^{1}$, \\ Wojciech Karlowski², Artur Jarmolowski ${ }^{1}$ and Zofia Szweykowska-Kulinska ${ }^{1}$
}

1Department of Gene Expression, Institute of Molecular Biology and Biotechnology, Faculty of Biology, Adam Mickiewicz University in Poznan, Poznań, Poland; 2Department of Computational Biology, Institute of Molecular Biology and Biotechnology, Faculty of Biology, Adam Mickiewicz University in Poznan, Poznań, Poland

\begin{abstract}
MicroRNAs are 19- to 24-nt-long single-stranded RNAs that are crucial regulators of gene expression which control plant development and response to environmental cues. We have analyzed microtranscriptomes of five barley developmental stages. Generally, during the barley development, miR168-3p and miR1432-5p levels increase while the 5'U-miR156-5p level decreases (with exception for the 2-week-old barley). We have identified two miR156-5p izomiRs (called 5'U-miR156-5p [20 nt] and 5'UU-miR156-5p [21 nt]), which were expressed differently during barley development. The 5' U-miR156$5 p$ level decreased in 3-week-, 6-week-, and 68-day-old barley, when compared to the 1-week-old plants. Meanwhile, the 5' UU-miR156-5p level increased significantly in the 68-day-old barley plants. Moreover, only the $5^{\prime}$ U-miR156 isomiR recognizes and guides unique transcription factor mRNAs from the Squamosa Promoter Binding Protein-Like (SPL) family. We identified many non-canonical microRNAs with changed expression levels during the barley development. Here, we present the profiles of microRNA expression characteristics for particular barley developmental stages. These analyses are accompanied by the experimental degradome analysis of miRNA targets.
\end{abstract}

Key words: barley, microRNA, deep sequencing, development

Received: 03 June, 2016; revised: 05 July, 2016; accepted: 08 September, 2016; available on-line: 02 November, 2016

\section{INTRODUCTION}

MicroRNAs are small molecules that act on complementary target mRNAs, leading to their degradation via the endonucleolytic activity of Argonaute (AGO). In Arabidopsis, there are ten AGO proteins (Garcia-Ruiz et al., 2015). AGO proteins that contain the DDH motif can cleave target mRNAs (Baumberger \& Baulcombe, 2005; Carbonell et al., 2012). A microRNA bound with AGO creates RISC (RNA-Induced Silencing Complex). Hsp90 facilitates RISC assembly (Iki et al., 2010). The role of microRNA is predominantly related to guiding the AGO1 protein to the proper mRNA target. Canonical cleavage of target mRNA occurs at the position located opposite the 10-11th nucleotide (counting from the guiding miRNA 5' end). In Arabidopsis, more than half of the target mRNAs are transcription factors (TFs) (Fahlgren et al., 2007). The primary transcript of a microRNA is produced by RNA Pol II followed by the excision of the pre-miR stem-loop precursor by the DCL1 in the nucleus. Then, double-stranded microRNA/ microRNA* duplex is excised from the pre-miR precursor, exported to the cytoplasm, and bound by the AGO protein (Mallory \& Vaucheret, 2010; Schirle \& MacRae, 2012). There are 325 precursors and 427 mature Arabidopsis microRNAs, and only 69 and 71 barley microRNA precursors and mature microRNAs (respectively) are deposited in the miRBase (MiRBase, release 21: June 2014; (Kozomara \& Griffiths-Jones, 2011)). The nucleotide sequence, miRNA length, 5' end terminal nucleotide of microRNAs, and $\mathrm{miR} / \mathrm{miR}^{*}$ duplex structure dictate the sorting and future action of a particular microRNA (Zhang et al., 2014). In Arabidopsis, a predominant fraction of microRNAs $(76 \%)$ contains molecules that are $21 \mathrm{nt}$ long, while the remnant microRNAs represent smaller populations: $11 \%-22$ nt long; 9\% - 20 nt long; $3 \%-24$ nt long; $1 \%-23$ nt long; and only $0.23 \%-19$ nt long. Additionally, in the case of $56 \%$ of $21 \mathrm{nt}$ Arabidopsis microRNAs, the first nucleotide from the 5' end is occupied by uridine, which suggests miRNA association with AGO1 and its involvement in mRNA cleavage (unpublished data, Zhang et al., 2014).

Barley development is mostly described by using phenotypical features like leaf number, tiller numbers, and kernel stage (Zadoks et al., 1974). In our previous work, we described pri-microRNA expression profiles in five barley developmental stages (Kruszka et al., 2013). Moreover, in the mirEX 2.0 database (an integrated environment for the expression profiling of plant microRNAs), we included 140 barley pri-microRNAs and also mature microRNA expression profiles for these 5 barley developmental stages (Zielezinski et al., 2015). Although the pri-miRNA and miRNA expression profiles are deposited in the mirEX 2.0 database, they were never analyzed and compared in detail in the different barley developmental stages. Here, we present a global analysis of the barley microtranscriptome profiles identifying the microRNAs specific for the various barley developmental stages. Moreover, we also identified miRNA-targeted mRNAs. In addition, we present a deeper microRNA isomiR analysis in the case of miR156-5p.

\section{MATERIALS AND METHODS}

Plant material. Hordeum vulgare cv. Rolap seeds were received from the Institute of Plant Genetics of the

e-mail: zofszwey@amu.edu.pl (ZSK); apacak@amu.edu.pl (AP) *These authors contributed equally to this work Abbreviations: NGS, Next Generation Sequencing 
Polish Academy of Sciences (Poznań, Poland) (Devaux et al., 1992). Plants were sampled in five developmental stages identified in the Zadoks scale (Zadoks et al., 1974): 1 -week-old plants (code 11, one leaf developed), 2-weekold plants (code 13, third leaf developed), 3-week-old plants (code 20-21, beginning of tillering), 6-week-old plants (code 32-36, stem elongation), and 68-day-old plants (code 75-77, kernels in milk ripeness). Ten whole plants for each particular developmental stage were treated as one sample.

RNA isolation. A total RNA enriched in small RNAs was isolated using the previously described approach (Kruszka et al., 2013). The quality of the isolated RNA was verified using an Agilent 2100 Bioanalyzer and Nano Plant RNA assay (Agilent).

Small RNA library preparation. Four independent small RNA libraries were designed for each barley developmental stage. Three replicas were performed using an Illumina TruSeq Small RNA Library Preparation Kit (Illumina). The quantification of libraries was carried out using a Quant-iT PicoGreen dsDNA Assay kit (Molecular Probes) reagent and Tecan Infinity 200 Pro Spectrometer (Tecan). NGS was performed using a HiScan-SQ machine (Illumina). The fourth replicated library for each developmental stage was performed using a different protocol. In brief, $1 \mu \mathrm{g}$ of total RNA was ligated to a $3^{\prime}$ adenylated adapter using a truncated T4 RNA ligase2 (New England Biolabs). After $1 \mathrm{~h}$ of incubation at $22^{\circ} \mathrm{C}$, an RNA 5' adapter was added with T4 RNA ligase (Ambion), ATP and incubated at $37^{\circ} \mathrm{C}$ for 1 hour. cDNA was synthesized using Superscript II Reverse Transcriptase (Invitrogen). PCR was performed using a Phusion Hot Start High Fidelity DNA polymerase (New England Biolabs). PCR was run at $98^{\circ} \mathrm{C}$ for $30 \mathrm{sec}$, followed by 15 cycles at $98^{\circ} \mathrm{C}$ for $15 \mathrm{sec}, 62^{\circ} \mathrm{C}$ for $30 \mathrm{sec}$, and $72^{\circ} \mathrm{C}$ for $15 \mathrm{sec}$, followed by a $5 \mathrm{~min}$ incubation. Products were separated electrophoretically, and the desired miRNA fraction in size was excised and eluted. The quality was tested using an Agilent Bioanalyzer DNA1000 chip and quantified using an Qubit fluorometer (Invitrogen). Each library was diluted to a target concentration for cluster generation and then loaded onto a lane of an Illumina GAiiX flow cell. The fourth library and its sequencing was performed at the $\mathrm{BC}$ Cancer Agency, Vancouver, BC, Canada.

Degradome. Degradome analysis (Parallel Analysis of RNA ends [PARE]) was performed using the approach used in previously published papers (German et al., 2009; Alaba et al., 2015).

Dataset analysis. Four replications were used for each particular barley developmental stage. Deep-sequencing data analysis was carried out using the approach described previously (Alaba et al., 2015). Then the normalized counts for each sRNA were averaged from replicates and the fold change and p-value were calculated between conditions using the Student's t-test. In the case of replications where no reads were detected (0 reads), value of 1 was added to the average in order to enable fold change calculation. For further analysis of the expression pattern, we considered microRNAs that have abundance described by either $\log _{2}$ (Fold Change) $>1$ (increase higher than 2 fold) or $\log _{2}$ (FC) $<-1$ (decrease higher than 2 fold) with the $p$ value $<0.05$ (Student's $t$-test). Barley pri-microRNA and microRNA expression profiles deposited in the mirEX 2.0 database are available at the following web page: http://www. combio.pl/mirex (Zielezinski et al., 2015).

Target prediction and verification based on the degradome data. Possible target identification was performed using the psRNATarget online tool, (http://
plantgrn.noble.org/psRNATarget) with default settings (Dai \& Zhao, 2011). When possible, the predicted targets were verified with our data obtained from the degradome sequencing.

Barley stem-loop pre-miR structure identification. For construction of the stem-loop pre-microRNA precursors, the following sequences were used:

- pre-miR827 - morex_contig_142720 CAJW010142720 carma $=2 \mathrm{HL}$

- pre-miR319-5p - morex_contig_46695 CAJW010046695 carma $=3 \mathrm{HS}$

- pre-miR171-5p - morex_contig_54459 CAJW010054459 carma $=2 \mathrm{HL}$

- pre-miR1432-5p - morex_contig_137798 CAJW010137798 carma $=2 \mathrm{HS}$

- pre-miR171-3p - morex_contig_2550634 CAJW012550634 carma $=2 \mathrm{HL}$

- pre-miR319-3p - morex_contig_134927 CAJW010134927 carma $=4 \mathrm{HS}$

- pre-miR156-5p - morex_contig_135129 CAJW010135129 carma $=6 \mathrm{HS}$

Sequences presented above were found by using the IPK BLAST Server (IPK Gatersleben - http:/ / webblast. ipk-gatersleben.de/barley/) and the ViroBLAST online tool (Deng et al., 2007). The obtained sequences were folded using Folder version 1.11 (RNAfold algorithm) software available at http://www.ncrnalab.dk/\#rnafolder/ rnafolder.php. The longest sequences which could form the stem-loop structures were used for pre-microRNA construction. The pre-microRNA structures with the lowest $\Delta \mathrm{G}$ energy value were chosen.

\section{RESULTS}

Using the Illumina deep-sequencing approach, we analyzed the microtranscriptomes of five barley developmental stages with regard to phenotypic traits. For each stage, four small independent RNA libraries were constructed and sequenced. Normalized reads from a particular developmental stage were compared, and fold changes were calculated. Analyses were performed in three ways:

(i) we identified the differences in microRNA expression profiles in 2-week-, 3-week-, 6-week-, and 68-dayold barley plants, when compared to the 1-week-old barley plants (Suppl. Table 1 at www.actabp.pl);

(ii) we compared microRNA expression profiles between particular barley stages to find unique expression profiles for the selected developmental stage (Suppl. Table 2 at www.actabp.pl);

Based on the statistically significant differentially expressed microRNAs presented in Suppl. Table 1, we analyzed 14 up-regulated and 11 down-regulated microRNAs in further steps. The same sets of microRNAs were analyzed for expression profiles in the barley developmental stages compared to 1 -week old barley plants (Fig. 1) and other barley developmental stages compared between each other (Fig. 2).

(iii) we analyzed potential target mRNAs for the selected microRNAs based on the performed degradome analysis. Degradome data were obtained for 68-day-old barley plants (Table 1). Characterization of the identified target mRNAs was carried out by comparisons to known nucleotide/protein sequences (Table 2).

\section{Barley microRNAs showing different expression profiles during development}

MiR168-3p and miR1432-5p are more frequent, and 5'U-miR156-5p (20 nt) is less frequent in all of the older 
Table 1. Barley microRNA targets. The targets were identified based on the degradome data obtained from PARE-Seq of 68-day-old barley.

Accession no, Target id - based on Ensembl Plants accession number; length, length of the analyzed cDNA; cleavage, cleavage_site (deg scoreldeg rank) - cleavage position, degradome score, degradome ranking; * cleavage position between nucleotides with match to 10 th and 11 th nucleotide of microRNA counting from the $5^{\prime}$ end, + non canonical cleavage position; score - aln score - alignment score between microRNA and target sequence (the lower the value, the better the score); energy, hybridization energy (kcal/mol). Only targets with the best score numbers were included in the table.

\begin{tabular}{|c|c|c|c|c|c|c|c|c|}
\hline microRNA & $5^{\prime}$ sequence $3^{\prime}$ & nt & Accession no & $\begin{array}{l}\text { length } \\
\text { (bp) }\end{array}$ & cleavage & non canonical & score & energy \\
\hline $\operatorname{miR} 156-5 p$ & ttgacagaagagagtgagcac & 21 & MLOC_52321.1 & 1607 & $\mathrm{c}: 871(1.54 \mid 9)^{*}$ & - & 2 & -34.4 \\
\hline $\operatorname{miR} 156-5 p$ & ttgacagaagagagtgagcac & 21 & MLOC_62426.1 & 1539 & c:933(0.78|11)* & $\mathrm{c:932(2.35|5)+}$ & 2 & -34.4 \\
\hline $\operatorname{miR} 156-5 p$ & ttgacagaagagagtgagcac & 21 & MLOC_11199.7 & 3162 & 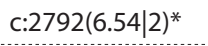 & - & 3 & -32.6 \\
\hline $\operatorname{miR} 156-5 p$ & ttgacagaagagagtgagcac & 21 & MLOC_13032.1 & 1112 & c:751(2.94|4)* & - & 4.5 & -31 \\
\hline $\operatorname{miR} 156-5 p$ & tgacagaagagagtgagcac & 20 & MLOC_37841.5 & 2319 & 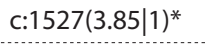 & - & 1.5 & -33.8 \\
\hline $\operatorname{miR} 156-5 p$ & tgacagaagagagtgagcac & 20 & MLOC_52321.1 & 1607 & $\mathrm{c}: 870(2.56 \mid 7)^{*}$ & c:871(1.54|9)+ & 1.5 & -33.8 \\
\hline miR156-5p & tgacagaagagagtgagcac & 20 & MLOC_62426.1 & 1539 & c:932(2.35|5)* & - & 1.5 & -34.1 \\
\hline miR156-5p & tgacagaagagagtgagcac & 20 & MLOC_61297.1 & 3239 & c:787(1.48|5)* & - & 1.5 & -33.8 \\
\hline miR156-5p & tgacagaagagagtgagcac & 20 & MLOC_11199.7 & 3162 & $\mathrm{c}: 2791(15.90 \mid 1)^{*}$ & $c: 2792(6.54 \mid 2)+$ & 3 & -31.7 \\
\hline $\operatorname{miR} 156-5 p$ & tgacagaagagagtgagcac & 20 & MLOC_13032.1 & 1112 & c:750(20.93|1)* & c:751(2.94|4)+ & 3 & -30.8 \\
\hline miR159-5p & gagctcctatcattccaatga & 21 & MLOC_38762.5 & 1004 & c:607(1.69|6)* & c:608(1.69|6)+ & 9 & -26.3 \\
\hline miR159-3p & tttggatgaagggagctctg & 20 & MLOC_75369.1 & 2856 & $\mathrm{c}: 1435(1.51 \mid 1)^{*}$ & - & 9.5 & -28.1 \\
\hline miR159-3p & tttggattgaagggagctctg & 21 & MLOC_55324.1 & 1336 & 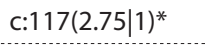 & - & 5.5 & -28.6 \\
\hline $\operatorname{miR} 159-3 p$ & tttggattgaagggagctctg & 21 & MLOC_71332.2 & 1877 & $\mathrm{c}: 863(3.67 \mid 2)^{*}$ & - & 6 & -32.5 \\
\hline miR159-3p & tttggattgaagggagctctg & 21 & MLOC_72581.1 & 1884 & 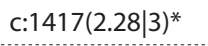 & - & 10 & -23.3 \\
\hline miR167-5p & tgaagctgccagcatgatctga & 22 & MLOC_58330.5 & 2656 & $\mathrm{c}: 2540(11.72 \mid 1)^{*}$ & - & 9.5 & -31.1 \\
\hline $\operatorname{miR} 168-5 p$ & tcgcttggtgcagatcgggac & 21 & MLOC_12447.2 & 3053 & $c: 249(2.52 \mid 3)^{*}$ & - & 8 & -33.9 \\
\hline miR168-3p & cccgccttgcaccaagtgaat & 21 & MLOC_63404.6 & 2457 & c:2226(2.60|8)* & c:2227(2.09|9)+ & 15 & -20.2 \\
\hline $\operatorname{miR} 169-3 p$ & ggcggtcaccttggctagc & 19 & MLOC_63790.1 & 2838 & 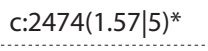 & - & 7 & -25.7 \\
\hline $\operatorname{miR} 171-5 p$ & cggtattggtgcggttcaatc & 21 & MLOC_11773.1 & 5489 & c:5188(1.68|7)* & - & 14.5 & -22.5 \\
\hline $\operatorname{miR319-5p}$ & agagcgtccttcagtccactc & 21 & MLOC_61362.3 & 1898 & c:1842(4.75|4)* & c:1843(4.31|5)+ & 13.5 & -24.2 \\
\hline miR319-3p & cttggactgaagggagctcc & 20 & MLOC_71332.2 & 1877 & 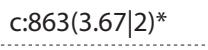 & - & 3.5 & -35.7 \\
\hline miR390-5p & aagctcaggagggatagcgсc & 21 & MLOC_37712.1 & 2369 & c:1889(2.14|7)* & - & 14.5 & -25.7 \\
\hline $\operatorname{miR390-5p}$ & aagctcaggagggatagcgcc & 21 & MLOC_33978.3 & 1949 & c:1652(10.17|2)* & - & 17.5 & -25.4 \\
\hline miR393-5p & ttccaaagggatcgcattgat & 21 & MLOC_56088.1 & 3166 & $\mathrm{c}: 2471(15.19 \mid 4)^{*}$ & - & 4.5 & -33.5 \\
\hline miR393-5p & ttccaaagggatcgcattgat & 21 & MLOC_9864.2 & 1973 & c:1429(7.27|6)* & - & 4.5 & -33.5 \\
\hline miR396-5p & tccacaggctttcttgaactg & 21 & MLOC_80060.1 & 1053 & c:753(9.59|1)* & - & 3 & -35.4 \\
\hline miR396-5p & tccacaggctttcttgaactg & 21 & MLOC_67201.2 & 2342 & c:913(13.91|1)* & - & 6.5 & -28.8 \\
\hline $\operatorname{miR} 408-3 p$ & $\operatorname{tgcactgcctcttccctggc}$ & 20 & MLOC_69288.1 & 1039 & c:861 $(8.16 \mid 1)^{*}$ & - & 1.5 & -39.8 \\
\hline $\operatorname{miR} 827-5 p$ & tttgttggttgtcatctaасс & 22 & MLOC_52144.1 & 2112 & c:1980(3.42|9)* & - & 12 & -16.4 \\
\hline $\operatorname{miR} 827-5 p$ & ttttgttggttgtcatctaacc & 22 & MLOC_70149.1 & 2853 & $\mathrm{c}: 1704(1.82 \mid 1)^{*}$ & - & 14 & -17.9 \\
\hline $\operatorname{miR} 827-5 p$ & tttgttggttgtcatctaасc & 22 & MLOC_59423.5 & 3157 & $\mathrm{c}: 1863(16.80 \mid 1)^{*}$ & - & 14.5 & -20.4 \\
\hline miR1432-5p & ttcaggagagatgacaccgac & 21 & MLOC_70272.1 & 1299 & c:295(7.20|1)* & - & 3.5 & -39.8 \\
\hline $\operatorname{miR} 1432-5 p$ & ttcaggagagatgacaccgac & 21 & MLOC_70788.1 & 800 & c:159(2.18|1)* & - & 3.5 & -35.7 \\
\hline miR1432-5p & ttcaggagagatgacaccgac & 21 & MLOC_61497.1 & 1339 & $\mathrm{c}: 1006(54.61 \mid 1)^{*}$ & - & 8.5 & -30.2 \\
\hline $\operatorname{miR} 1432-5 p$ & tcaggagagatgacaccgac & 20 & MLOC_70788.1 & 800 & - & c:159(2.18|1)+ & 2 & -35.5 \\
\hline $\operatorname{miR} 1432-5 p$ & tcaggagagatgacaccgac & 20 & MLOC_70272.1 & 1299 & - & $\mathrm{c:295(7.20|1)+}$ & 4 & -39.6 \\
\hline $\operatorname{miR} 1432-5 p$ & tcaggagagatgacaccgac & 20 & MLOC_61497.1 & 1339 & $\mathrm{c}: 1005(14.12 \mid 3)^{*}$ & $\mathrm{c}: 1006(54.61 \mid 1)+$ & 5 & -30.2 \\
\hline $\operatorname{miR} 6201-5 p$ & tgaccctgaggcactcataccg & 22 & MLOC_52074.1 & 653 & c:209(2.01|10)* & $c: 210(2.01 \mid 10)+$ & 13.5 & -26.2 \\
\hline
\end{tabular}

barley stages (except for 5'U-miR156-5p in 2-week old barley), when compared to the 1-week stage (Fig. 1A-H, Suppl. Table 1). MiR168-3p was earlier considered as a passen- ger miRNA for miR168-5p. MiR168-3p has a higher abundance level at all stages when compared to the 1-week-old barley plants, with the highest 2.8 fold change in 2 -week- 

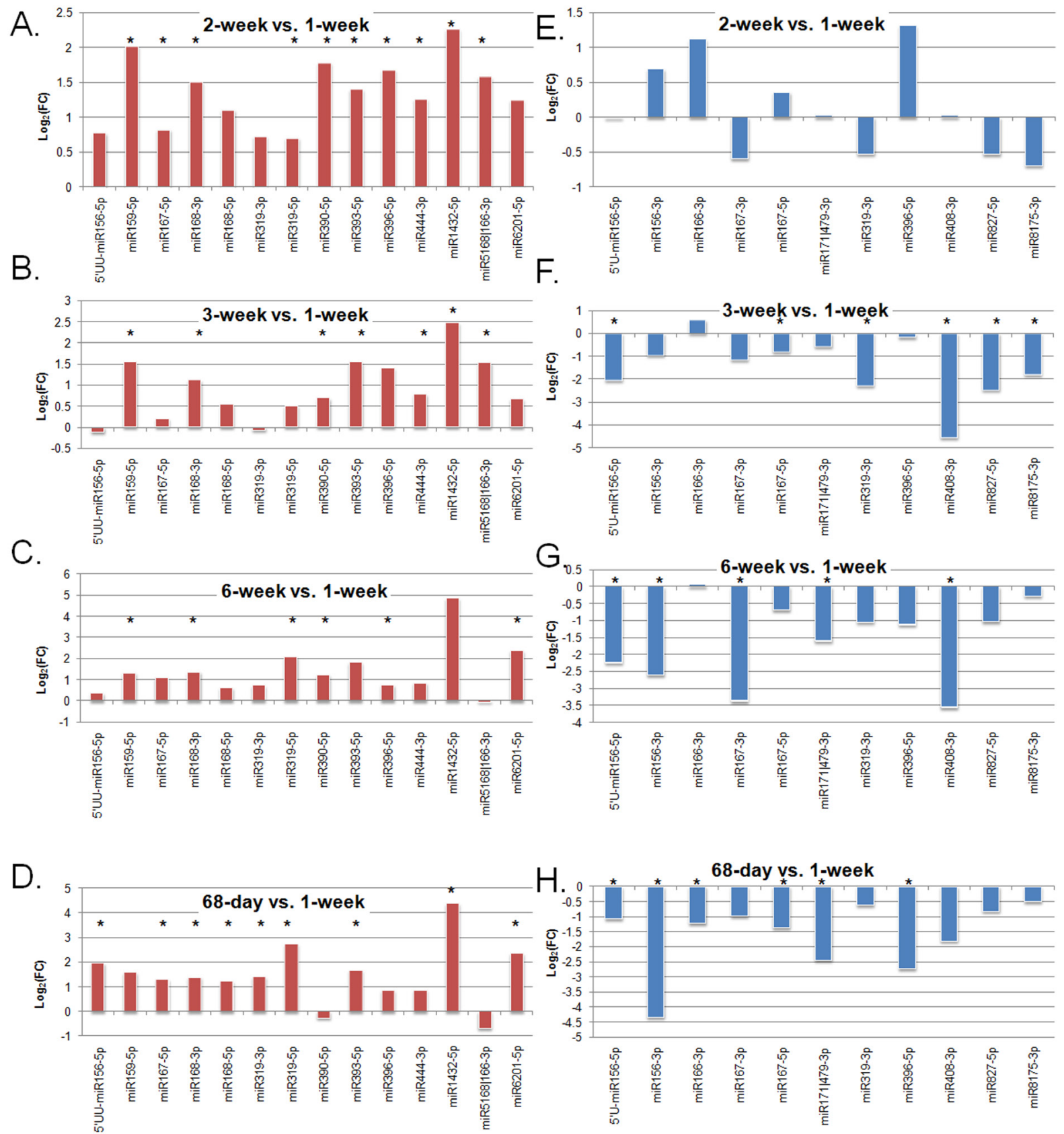

Figure 1. Fold change of microRNA expression in:

(A) 2- vs. 1-week-old barley; (B) 3-vs. 1-week-old barley; (C) 6- vs. 1-week-old barley; (D) 68-day- vs. 1-week-old barley; (E) 2- vs. 1-weekold barley; (F) 3-vs. 1-week-old barley; (G) 6-vs. 1-week-old barley; and (H) 68-day- vs. 1-week-old barley. Red and blue bars represent the elevated and decreased microRNA levels, respectively. Fold changes a(re represented as $\log _{2}(\mathrm{FC})$ values. Symbol * depicts $p$-value $<0.05$. Detailed information about presented microRNA is included in Suppl. Table 1.

old plants (Fig. 1A-D). The degradome-approved mRNA target for this miRNA encodes the regulator of chromosome condensation (RCC1) protein (Table 2).

The level of miR168-5p (21 nt) was only significantly increased in 68-day-old barley plants (Fig. 1A-D). Arabidopsis miR168-5p targets Ago1 mRNA; thus, it controls the post-transcriptional gene silencing process. We also found miR168-5p guided Ago1 mRNA cleavage fragments in the barley degradome data. (Table 1, Table 2).

MiR1432-5p levels highly increased in 6-week- and 68-day-old barley, which targeted mRNAs encoding protein possessing EF-hand, calcium binding motif, and protein with 2-oxoglutarate (2OG) and Fe(II)-dependent oxygenase domain (Figs. 1C-D; Fig. 2B; Table 1, Table 2, Suppl. Table 2 at www.actabp.pl). The obtained pre-miR1432 structure shows the presence of a properly folded stem-loop (Fig. 3A).

MiR156-5p is a highly abundant microRNA in all barley developmental stages. In rice, there are 12 miR156-5p species (a-1), and all of them target SPL TF mRNAs. The most abundant 20 nt 5'U-miR156-5p (5' TGACAGAAGAGAGTGAGCAC 3') molecule is present in 1- and 2-weekold barley. In older stages, the level of this microRNA is much lower (Figs. 1F-H, Fig. 2D). The target mRNAs identified in the barley degradome data encode six TFs (Table 1, Table 2). MiR159-5p expression was significantly 
3-week vs. 2-week
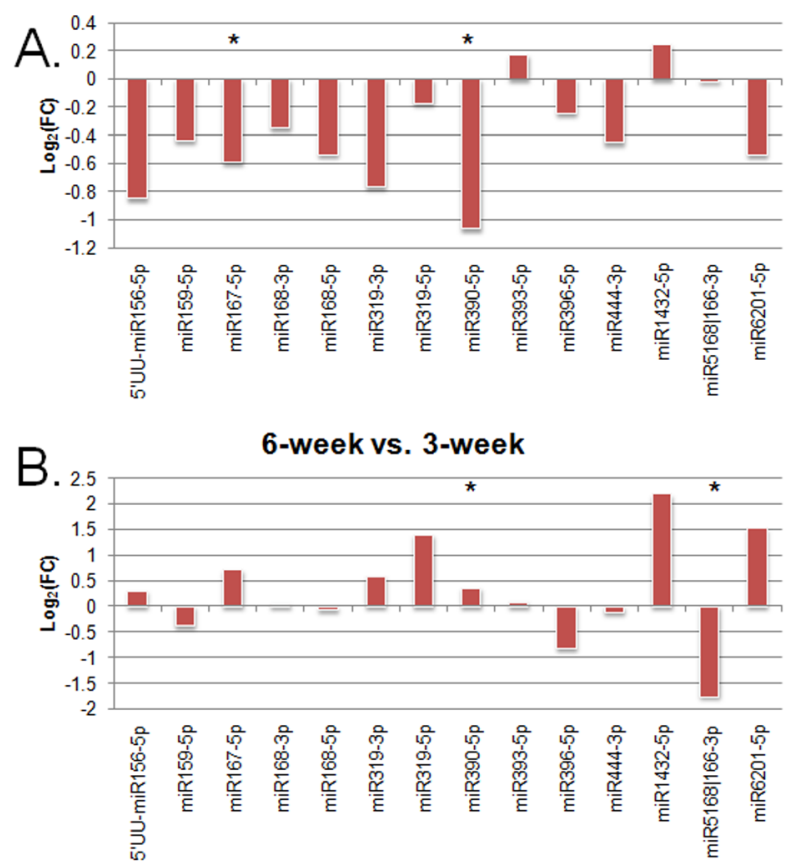

C.

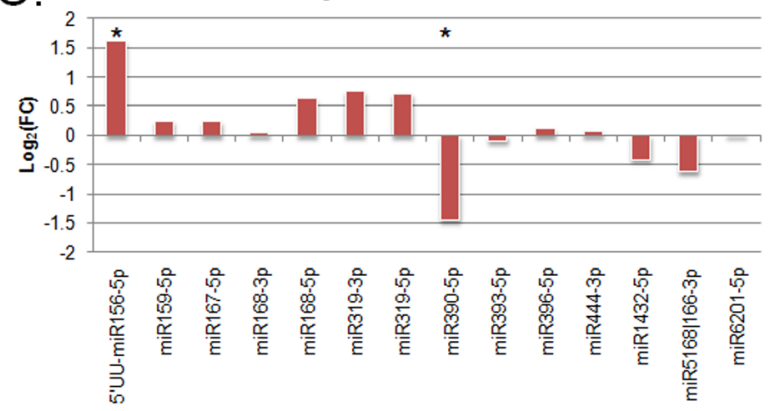

3-week vs. 2-week

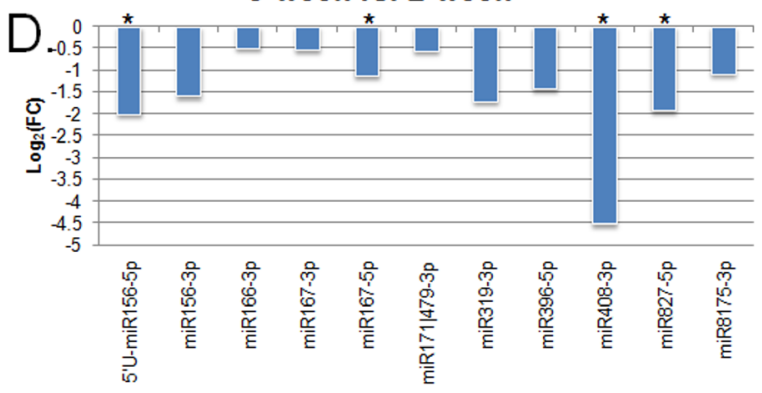

6-week vs. 3-week

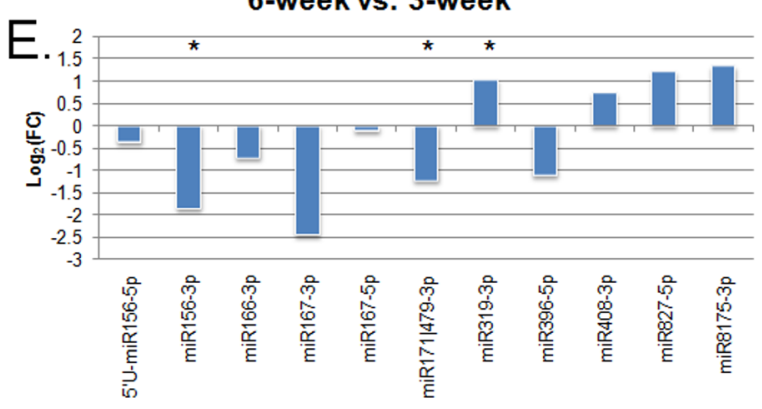

F.

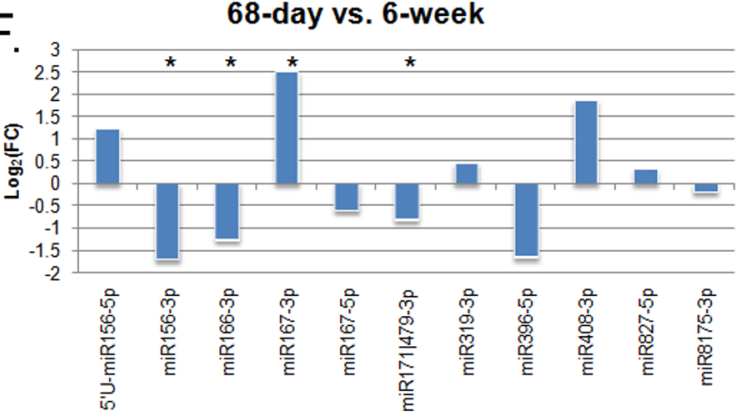

Figure 2. Fold change of microRNAs expression in:

(A) 3- vs. 2-week-old barley; (B) 6- vs. 3-week-old barley; (C) 68-day-vs. 6-week-old barley; (D) 3-vs. 2-week-old barley; (E) 6- vs. 3-weekold barley; and (F) 68-day-vs. 6-week-old barley. Red and blue bars on the charts represent the same set of microRNA molecules which were analyzed in Fig. 1. Symbol * depicts $p$-value $<0.05$.

higher in 2-, 3-, and 6-week-old barley plants; however, in 68-day-old barley, its expression increased by $\log _{2}(\mathrm{FC})=1.58$, but not significantly (Figs. 1A-D, Suppl. Table 1 at www. actabp.pl). The degradome data identified its mRNA target encoding pyruvate decarboxylase (Table 1, Table 2). However, in the 68-day developmental stage, we observed an increased level of the miR159-3p isomiR (Suppl. Table 1 at www.actabp.pl). In the degradome data we found target for this miRNA; i.e., an mRNA encoding LIM-domain binding protein (Table 2). In 68-day-old barley, we identified another miR159-3p isomiR that is more abundant than the previous one, and it was expressed higher in the 68-day-old barley than in 1-week-old barley (but not significantly). With the degradome data, we identified its target mRNA - MYB TF (Table 1, Table 2).

\section{MicroRNA expression level as a specific marker for a given developmental stage}

Comparison of microRNA expression in 2- versus 1-week-old barley plants.

It is known that leaves and new tillers start to develop in the 2-week-old barley plants. At this developmental stage, the level of the following eleven microRNAs is significantly increased: miR156-5p, miR159-5p, miR1685p/-3p, miR172-5p, miR390-5p, miR393-5p, miR396-5p, miR444-3p, miR528-3p, miR1432-5p, and miR5168-3p (Fig. 1A, Suppl. Table 1 at www.actabp.pl). Some of these microRNAs are directly related to the barley development. For reference, miR393-5p targets mRNAs that encode Transport Inhibitor Response1 (TIR1) and Auxin Signaling F box 2 and 3 proteins (AFB 2, AFB3) involved in the leaf development (Si-Ammour et al., 2011). MiR396 targets GRF1 (Growth-Regulating Factor 1) and GRF9 mRNA (Wang et al., 2011). In Arabidopsis, AtGRF1 TF is required for the coordination of cell division and differentiation during leaf development (Wang et al., 2011). Since miR396-5p is the most-abundant microRNA (Fig. 1A, Suppl. Table 1 at www.actabp. $\mathrm{pl}$ ), this may suggest the elevated importance of miR396$5 \mathrm{p}$ at the 2-week-old barley developmental stage. With the degradome data, we identified their mRNA targets as TIR1, AFB, and GRF (Table 1, Table 2). As mentioned earlier miR159-3p targets MYB TF mRNA (Allen et al., 2010). We observed that miR159-5p is at a higher level in the 2-week-old barely (Fig. 1A). Degradome analysis revealed target mRNA encoding pyruvate decarboxylase 
Table 2. Identification of the predicted target.

Accession number - Ensembl Plants database, GenBank database.

\begin{tabular}{|c|c|c|}
\hline microRNA & Accession number & Protein function \\
\hline miR156-5p & MLOC_61297.1 & Squamosa promoter-binding-like protein, BAJ94319.1 \\
\hline miR156-5p & MLOC_37841.5 & Squamosa promoter-binding-like protein, BAK05794.1 \\
\hline $\operatorname{miR} 156-5 p$ & MLOC_52321.1 & Squamosa promoter-binding-like protein, BAJ87295.1 \\
\hline miR156-5p & MLOC_11199.7 & Squamosa promoter-binding-like protein, BAJ97637.1 \\
\hline $\operatorname{miR} 156-5 p$ & MLOC_13032.1 & Squamosa promoter-binding-like protein \\
\hline $\operatorname{miR} 156-5 p$ & MLOC_62426.1 & Squamosa promoter-binding-like protein, BAJ92814 \\
\hline $\operatorname{miR} 159-5 p$ & MLOC_38762.5 & Pyruvate decarboxylase \\
\hline miR159-3p & MLOC_55324.1 & uncharacterized protein \\
\hline $\operatorname{miR} 159-3 p$ & MLOC_71332.2 & MYB TF \\
\hline miR159-3p & MLOC_72581.1 & TCP TF \\
\hline miR159-3p & MLOC_75369.1 & LIM-domain binding protein \\
\hline $\operatorname{miR} 167-5 p$ & MLOC_58330.5 & Auxin response factor (ARF) \\
\hline $\operatorname{miR} 168-5 p$ & MLOC_12447.2 & Argonaute 1 \\
\hline $\operatorname{miR} 168-3 p$ & MLOC_63404.6 & Regulator of chromosome condensation (RCC1) family protein \\
\hline miR169-3p & MLOC_63790.1 & Serine/Threonine-protein kinase \\
\hline $\operatorname{miR} 171-5 p$ & MLOC_11773.1 & Alpha, alpha-trehalose-phosphate synthase (UDP-forming) \\
\hline $\operatorname{miR3} 19-5 p$ & MLOC_61362.3 & CBL-interacting protein kinase 23 \\
\hline $\operatorname{miR319-3p}$ & MLOC_71332.2 & MYB TF \\
\hline $\operatorname{miR} 390-5 p$ & MLOC_37712.1 & GRAS (GAl, RGA, SCR) protein family, chitin-inducible gibberellin-responsive protein 1 \\
\hline $\operatorname{miR} 390-5 p$ & MLOC_33978.3 & Homeobox-leucine zipper protein HOX32 \\
\hline $\operatorname{miR393-5p}$ & MLOC_56088.1 & Auxin Signaling F-BOX \\
\hline $\operatorname{miR393-5p}$ & MLOC_9864.2 & Transport Inhibitor Response 1 \\
\hline $\operatorname{miR} 396-5 p$ & MLOC_80060.1 & Growth-Regulating Factor 9, GRF9 \\
\hline miR396-5p & MLOC_67201.2 & Growth-Regulating Factor 2 \\
\hline miR408-3p & MLOC_69288.1 & Blue copper protein \\
\hline $\operatorname{miR} 827-5 p$ & MLOC_52144.1 & RNA helicase \\
\hline $\operatorname{miR} 827-5 p$ & MLOC_70149.1 & SRG1 protein \\
\hline $\operatorname{miR} 827-5 p$ & MLOC_59423.5 & protein with DUF1668 domain \\
\hline miR1432-5p & MLOC_70272.1 & EF-hand, calcium binding motif \\
\hline $\operatorname{miR} 1432-5 p$ & MLOC_70788.1 & EF-hand, calcium binding motif \\
\hline miR1432-5p & MLOC_61497.1 & $\begin{array}{l}\text { Naringenin, 2-oxoglutarate 3-dioxygenase, 2-oxoglutarate (2OG) and Fe(II)-dependent oxygenase } \\
\text { superfamily protein }\end{array}$ \\
\hline miR6201-5p & MLOC_52074.1 & $66 \mathrm{AA}$ protein with uncharacterized function \\
\hline
\end{tabular}

for this miRNA (Table 1, Table 2). MiR528-3p has the second highest up-regulation level (8.6 fold change in comparison to 1 -week-old plants) of all of the up-regulated miRNAs at this developmental stage (Suppl. Table 1 at www.actabp.pl). We also identified and folded its pre-miR stem-loop structure (Fig. 3B). Since we did not identify any target for this miRNA in the degradome data, we used psRNATarget software to predict a putative one (Dai \& Zhao, 2011). We identified two potential targets of the miR528-3p, described as Caleosin 1 (Clo1, acc BI949541, translational inhibition) and aconitate hydratase mRNA (acc BI952160, mRNA cleavage).

Comparison of microRNA expression in 3-versus 1-week-old barley plants.

A 3-week-old barley plant develops new tillers. Among eight significantly up-regulated microRNAs, the highest expression in the 3-week old barley was detected for miR168-3p, miR159-5p and miR393-5p (Fig. 1B, Suppl. Table 1 at www.actabp.pl). Eight microRNA species have significantly lower levels when compared to the 1-week-old barley plants: miR156-5p/-3p, miR1675p, miR169-5p/-3p, miR319-3p, miR408-5p/-3p, miR827-5p, miR5072-5p (16 nt) and miR8175-3p (Suppl. Table 1 at www.actabp.pl). In the cases of miR156-5p and miR408-3p, we observed a rapid down-regulation of their levels. The degradome analysis points to the mRNA target for miR408-3p that encodes the Blue copper protein mRNA (Table 1, Table 2). MiR167-5p was up-regulated in the 3-week-old barley plants (Suppl. Table 1 at www.actabp.pl). Interestingly, we found the highest level of canonical 21-nt-long miR167-5p in the 3-week-old barley plants; however, two different 22-ntlong miR167-5p isomiRs have an even higher abundance 
Table 3. The average normalized reads of miR167-5p in five barley developmental stages.

\begin{tabular}{|c|c|c|c|c|c|c|}
\hline miR167-5p & length, bp & 1-week & 2-week & 3-week & 6-week & 68-day \\
\hline tgaagctgccagcatgatctg & 21 & 45 & 81 & 121 & 93 & 106 \\
\hline tgaagctgccagcatgatctgc & 22 & 3011 & 6462 & 6191 & 4349 & 3415 \\
\hline tgaagctgccagcatgatctga & 22 & 1718 & 3243 & 2112 & 3990 & 4231 \\
\hline tgaagctgccagcatgatctgg & 22 & 10 & 23 & 15 & 22 & 31 \\
\hline
\end{tabular}

A.

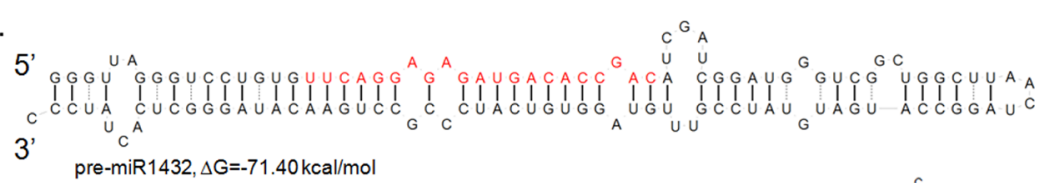

B.

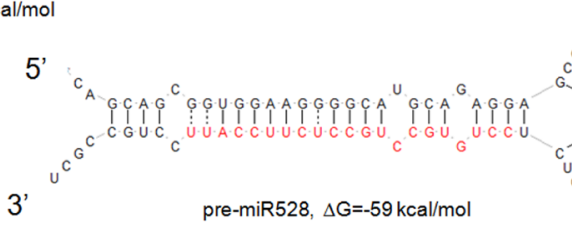

C.

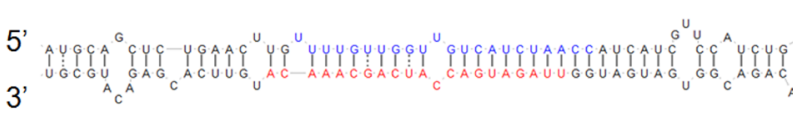
pre-miR827, $\Delta \mathrm{G}=-94.10 \mathrm{kcal} / \mathrm{mol}$

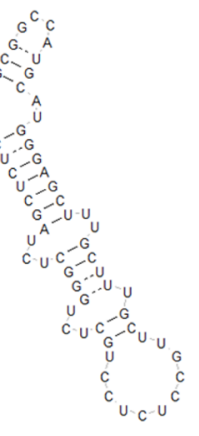
3
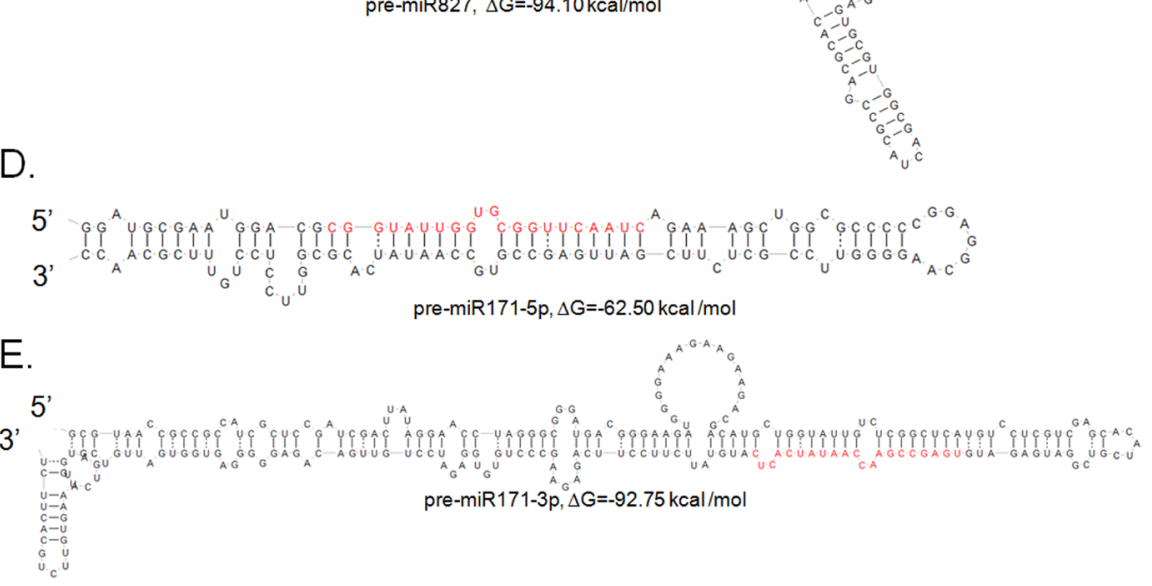

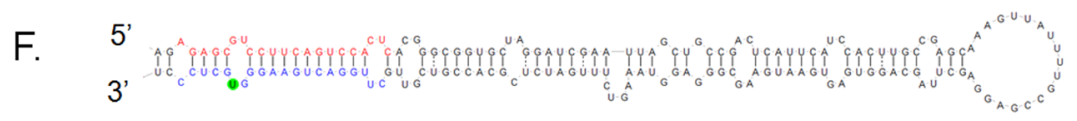

pre-miR319-5p, $\Delta G=-90.21 \mathrm{kcal} / \mathrm{mol}$

G.

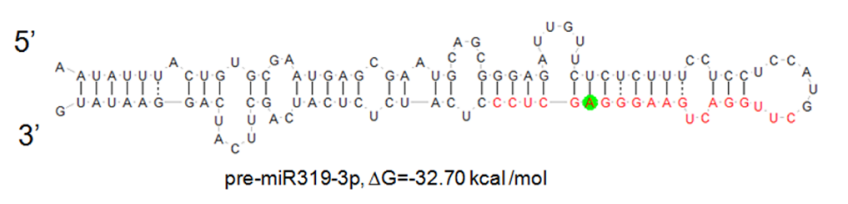

H. 5'

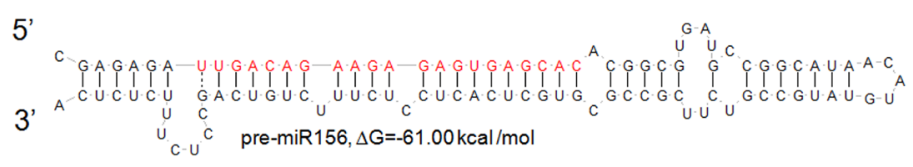

Figure 3. Stem-loop structures of the selected barley pre-miRNAs:

(A) pre-miR1432; (B) pre-miR528; (C) pre-miR827; (D) pre-miR171-5p; (E) pre-miR171-3p; (F) pre-miR-319-5p; (G) pre-miR319-3p; and (H) pre-miR156 for 5'UU-miR156-5p. Red and blue letters represent microRNA or microRNA*/non-canonical microRNA sequence, respectively. Structures with the lowest free energy are presented. 
A.

MLOC_11199.7, 5' GAUCUUCAGCAUGCUCUCUCÚ'CU CUGUCAAGCAACCCAGUGGGUGCUGC 3'

3' CACGAGUGAGAGAAGACAGU 5', 5'U-miR156-5p

3' CACGAGUGAGA' ${ }^{*}$ *AAGACAGUU 5', 5'UU-miR156-5p

MLOC_13032.1, 5' CAGAUAAACCUACCUAAGCAUGCUCCCUCÚCUUCUGUCAUGCAGCCAUGC 3’'

MLOC_37841.5, 5' CGACUCGGACUGUGCUCUCUCUCUUCUGUCAGCUCCGGCAAAUUCCUCCG 3'

3' CACGAGUGAGAGAAGACAGU 5' 5’U-miR156-5p

B.

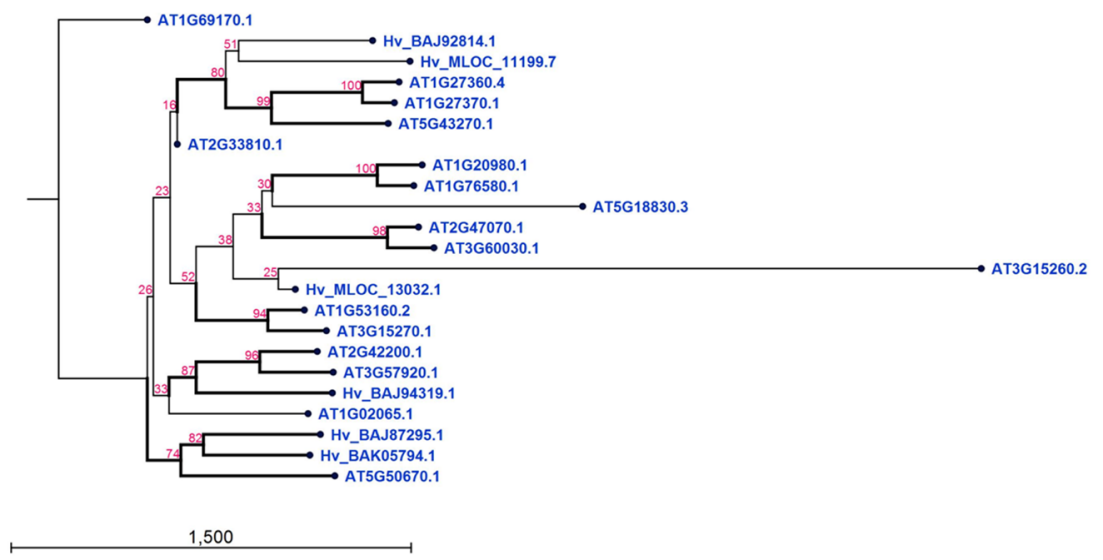

Figure 4. (A) The cleavage sites in MLOC_11199.7, MLOC_13032.1, MLOC_37841.5 and MLOC_61297.1 targeted by 5'UU-miR156-5p and/or 5'U-miR156-5p. (B) Phenogram of Arabidopsis SPL TF amino-acids sequences and six barley SPL TFs.

Barley MLOC_11199.7, MLOC_13032.1, MLOC_52321.1, MLOC_62426.1 mRNA are targeted by 5'UU-miR156-5p and 5'U-miR156-5p; MLOC_37841.5, MLOC_61297.1 mRNA are targeted exclusively by 5' U-miR156-5p. The tree was constructed using N-J algorithm, bootstrap $=1000$ replication, CLC Main Workbench (Qiagen) software. Arabidopsis thaliana SPL TFs accession numbers: AT2G47070.1 SPL1; AT5G43270.1 - SPL2; AT2G33810.1 - SPL3; AT1G53160.2 - SPL4, AT3G15270.1 - SPL5, AT1G69170.1 - SPL6; AT5G18830.3 - SPL7; AT1G02065 - SPL8; AT2G42200.1 - SPL9; AT1G27370.1 - SPL10; AT1G27360 - SPL11; AT3G60030.1 - SPL12; AT5G50670.1 - SPL13B; AT1G20980.1 - SPL14; AT3G57920.1 - SPL15; AT1G76580.1 - SPL16, Barley SPL TF accession numbers: MLOC_11199.7, MLOC_13032.1, BAJ87295 (MLOC_52321.1), BAJ92814 (MLOC_62426.1), BAK05794 (MLOC_37841.5), BAJ94319 (MLOC_61297.1).

(Table 3). MiR167-5p targets ARF6 and ARF8 (Auxin Response Factor) mRNAs (Liu et al., 2014) and in barley also NEK5-like kinase (Kruszka et al., 2014). In the degradome data, we also found $A R F$ mRNA targeted by miR167-5p. MiR827-3p expression is induced by phosphate starvation (Hackenberg et al., 2013). In Arabidopsis, this microRNA targets NLA mRNA (Nitrogen Limitation Associated) that encodes the E3 ubiquitin ligase involved in Pi-related protein degradation via the proteasome pathway. In rice and barley, miR827-3p targets the SPX-MFS mRNA-encoding protein involved in $\mathrm{Pi}$ sensing and transport (Lin et al., 2010; Hackenberg et al., 2013). Interestingly, we also identified miR827-5p that was expressed at a lower level in the 3-week-old barley plants (Fig. 1F). MiR827-5p and miR827-3p are derived from the same precursor (Fig. 3C). Based on the degra- dome analysis, we identified several mRNA targets that are potentially targeted by miR827-5p (Table 1, Table 2).

Comparison of microRNA expression in 6-versus 1-week-old barley plants.

The 6-week-old barley plant elongates its shoots and is still before its flowering time. The highest expression levels were recorded for the following microRNAs: miR168-3p, miR159-5p, miR319-5p, miR6201-5p, miR390-5p, and 20 nt miR1432-5p isomiR (Fig. 1C, Suppl. Table 1 at www.actabp.pl). Interestingly the highest abundance of $20 \mathrm{nt}$ and $21 \mathrm{nt}$ miR1432-5p isomiRs is specific for this stage (Suppl. Table 1 at www.actabp. $\mathrm{pl}$. Moreover, up-regulation of miR390-5p is observed (Fig. 1C, Fig. 2B, 2C), although the highest miR390-5p level fold change is observed for 2 -week vs. 1-week-old 
Table 4. MiR156 with significant expression fold change in 68-day- vs. 1-week-old barley.

\begin{tabular}{lllll}
\hline miR & sequence & $\begin{array}{c}\text { average reads } \\
\text { no (68-day old) }\end{array}$ & $\log _{2}(\mathrm{FC})$ & $p$-value \\
\hline 5'UU-miR156-5p & ttgacagaagagagtgagcac & 12749.3525 & 1.96 & 0.001116 \\
\hline 5'U-miR156-5p & tgacagaagagagtgagcac & 10157.55 & -1.07 & 0.022749 \\
miR156-5p & tgacagaagagagtgagtac & 8.8475 & -2.7 & 0.045473 \\
miR156-5p & acagaagagagtgagcaca & 0.565 & -2.99 & 0.027137 \\
miR156-5p & tgacagaagagagtgagcaca & 16.9375 & -2.92 & 0.036196 \\
\hline miR156-3p & gctcactgctctatctgtcacc & 5.04 & -4.35 & 0.004855 \\
\hline
\end{tabular}

barley (Fig. 1A). It is known that Arabidopsis miR390-5p is recruited by an AGO7 and targets TAS3 RNA (Fahlgren et al., 2006). As a consequence, ta-siRNAs are produced. In the subsequent steps, ta-siRNAs target $A R F-2$, $A R F-3$, and $A R F-4$ mRNAs, which control lateral root growth (Marin et al., 2010; Endo et al., 2013). We did not find a barley TAS3 homologue in either the Ensemble Plants or psRNATarget databases. However, the barley degradome analysis allowed us to find other genes, described as MLOC_37712.1 - belonging to the GRAS (GAI, RGA, SCR) protein family, chitin-inducible gibberellin-responsive protein 1, and MLOC_33978.3 encoding Homeobox-leucine zipper protein (HOX32), as targets for miR390-5p. The highly expressed miR1432-5 targets the mRNAs that encode the 2-oxoglutarate 3-dioxygenase protein as well as the protein with EF-hand, calcium-binding motifs (Table 1, Table 2).

Two microRNAs (miR171-5p and miR319-5p) exhibit elevated expression in the 6-week-old barley plants; however, their counterparts (miR171-3p and miR319-3p) show concomitantly lower expression levels (Suppl. Table 1 at www.actbp.pl). Rice miR171c-3p targets four GRAS (GAI-RGA-SCR) plant-specific transcription factors mRNA (Fan et al., 2015). PsRNATarget analyses revealed another mRNA target for the barley miR171-3p encoding the LRR receptor-like serine/threonine-protein kinase (TC239462). The target sequence for the miR319-5p was identified as CBL-interacting protein kinase 23 mRNA, for the 20-nt-long miR319-3p the mRNA target encodes the MYB TF (Table 1, Table 2). The miR319-5p is $21 \mathrm{nt}$ in length, is expressed at a high level, and is more abundant than the 20-nt miR319-3p that is down-regulated. Interestingly, we found that mentioned above miR171-5p/miR171-3p, and miR319-5p/miR319-3p are not derived from the same precursors (Fig. 3D-G).

Comparison of microRNA expression in 68-day- versus 1-weekold barley plants.

The 68-day-old barley plants have already developed spikes with kernels at the milk stage. Thirteen microRNAs were identified as having significantly higher levels; among them are: miR156-5p (5'UU - 21 nt), miR168-5p, miR319-3p, miR167-5p, miR393-5p, miR1432-5p, miR6201-5p. In contrast to all of the earlier developmental stages, the isomiR 5'UU-miR156-5p exhibited a uniquely higher level when compared to the 1 -week-old plants (Fig. 1D). This isomiR is identical to Glycine $\max \operatorname{miR} 156 \mathrm{k} / \mathrm{n} / \mathrm{o}$ isomiRs and can be diced out from the pre-miR156 precursor (Fig. 3H). On the other hand, the 5'U-miR156-5p was down-regulated (Fig. 1H, Table 4). We analysed six SPL mRNA targets of the miR156-5p isomiRs and found that the four mRNA targets can be potentially recognized by both, the 5'UUand 5'U-miR156-5p (21 nt and $20 \mathrm{nt}$ ) isomiRs, but two other targets can be recognized exclusively by the 5'U-miR156-5p (Fig. 4A, Table 1, Table 2). Moreover, comparison with the known Arabidopsis SPL TFs showed that the barley targets belong to a different SPL TF class (Fig. 4B). SPL TF encoded by the MLOC_612971 was targeted by the 5 ' U-miR 156-5p and is similar to Arabidopsis SPL9 and SPL15 TFs.

MiR1432-5p, miR6201-5p are monocot specific (Schreiber et al., 2011; Pandey et al., 2014). They are especially expressed in the 6-weekand 68-day-old barley plants. MiR6201 was predicted to have one mRNA target in wheat (Pandey et al., 2014), encoding a protein similar to proteinase inhibitor Rgpi9 (Pandey et al., 2014). In the degradome data, we found one target (MLOC_52074.1) that encodes a short 66 AA peptide of unknown function.

\section{DISCUSSION}

Deep sequencing of small RNAs is a powerful tool to discriminate between microRNA sequences and their abundance (Sobkowiak et al., 2012; Lukasik et al., 2013; Barciszewska-Pacak et al., 2015). Analysis of the changes in small RNA level between the particular developmental stages of barley plants revealed some interesting data, of which two observations seem to be the most profound: (i) the 20-nt 5'U-miR156-5p level decreases during barley development, while in spike (68-day-old plants), upregulation of the 21-nt 5'UU-miR156-5p is observed. Both isomiRs target the same mRNAs, but the shorter one additionally guides the cleavage of two unique mRNAs; (ii) the level of miR390-5p was specifically elevated only in the 2-week and 6-week-old plants (more than 2 fold). Some interesting observations concern the microRNA and its microRNA* levels. Previously, we identified miR159b-3p as an up-regulated microRNA during barley development (Kruszka et al., 2013). Here, we found that, apart from the miR159b-3p increase, the highest fold change is observed for its microRNA* - miR159b5p. MiR168-3p is up-regulated at all stages as compared to the 1-week-old barley, while the 21 nt miR168-5p is significantly up-regulated only in the 68-day-old barley plants. Our previous work had shown that the level of miR168-5p was almost unchanged and the level of miR168-3p was even decreased when compared to the 1-week barley stage (Kruszka et al., 2013). However, the previous analysis was based on northern blot hybridization, which is less sensitive and often does not discriminate between microRNAs belonging to the same family. Thus, sRNA deep sequencing enables a much-deeper analysis of microRNAs derived from the same family or isomiRs.

It is known that the miR156-5p expression profile is the opposite of the miR172 expression pattern. In rice, miR156 targets 11 SPL genes (Xie et al., 2006; Xie et al., 2012). SPL TFs regulate miR172 expression (Spanudakis \& Jackson, 2014). It is possible that the same regulation occurs in barley plants. In further studies, it will be interesting to establish which miR156-5p isomiR affects the miR172 expression in barley. As previously mentioned, 5'U-miR156-5p $20 \mathrm{nt}$ is down-regulated, but its 21-nt isomiR with additional $U$ residue at the 5' end is 
up-regulated in the 68-day-old barley when compared to 1 -week-old plants. One additional nucleotide at the 5'end of the miR156-5p allows for the recognition of different SPL mRNAs. In the degradome dataset, we found indeed that different SPL mRNAs are targeted by these two isomiRs of the miR156-5p. Comparison with known Arabidopsis SPL TFs showed that SPL mRNA that are targets for miR156-5p belong to different SPL classes. In agreement with these observations, we found that 20-nt 5'U-miR156-5p recognizes SPL mRNAs similar to Arabidopsis SPL9 and SPL15 TF mRNAs (Fig. 4B). Thus, microRNA isomiRs derived from a single pri-miRNA can specifically regulate various SPL gene expression. It will be interesting to find an exact relationship between the up- and down-regulated miR156-5p isomiRs and their SPL mRNA targets. The deep-sequencing data of sRNAs isolated from plants from several developmental stages confirm earlier observations published in our previous paper (Kruszka et al., 2013): Northern blot hybridization showed the presence of two microRNAs156-5p that differed in size. The northern signals in the 68-day-old barley reflect up-regulated 21-nt-long 5'UU-miR156-5p. In the mirEX2.0 database, there are expression profiles of three barley miR156 family members: miR156b, miR156c, and miR156d. These three microRNA156a/b/c are downregulated in the 6-weekand 68-day-old barley plants. This reflects the decreased expression of 5'U-miR156-5p. MiR156 expression decreased in shoots or whole rice plants; however, careful inspection of selected organs revealed that this microRNA level is increased in older leaves (Xie et al., 2012). The authors did not discriminate between the isomiRs. Here, we have shown that, in barley plants at the spike-formation stage (68-day-old plants), there are two miR156-5p izomiRs, which are differentially expressed.

Compared to the 6-week-old plants, we observed a decreased level of miR390-5p in the 68-day-old barley plants. This microRNA regulates the production of Arabidopsis ta-siRNAs that, in turn, downregulate ARF2, ARF3, and ARF4 TF levels. It was shown that the decreased level of ARF2, ARF3, and ARF4 TFs promotes lateral root growth (Marin et al., 2010). It would be interesting to test the level of these transcription factors in the 68-day-old barley plants and during lateral root growth. One of the elevated microRNAs in older barley plants is the miR1432-5p that targets the mRNA-encoding protein containing 2-oxoglutarate (2OG) and Fe(II)dependent oxygenase domains. We blasted the barley MLOC_61497.1 (target of the miR1432-5p) to the NCBI protein database. The highest similarity to this barley sequence was found in the case of Naringenin, 2-oxoglutarate 3-dioxygenase from Triticum urartu (EMS57783.1, $\mathrm{E}$ value $=0.0$, identities $=95 \%, 323 / 339)$. This enzyme catalyzes the 3-beta-hydroxylation of 2S-flavanones to 2R, 3R-dihydroflavonols, which are intermediates in the biosynthesis of flavonols, anthocyanidins, catechins, and proanthocyanidins. Altogether, we observed that during barley development, microRNA expression profiles change qualitatively and quantitatively. These changes influence the mRNA target levels and plant development.

\section{Acknowledgements}

Deep sequencing (three replicas) was performed at the Genome Analysis Laboratory (IBMiB, Faculty of Biology at the Adam Mickiewicz University in Poznan) funded by National Multidisciplinary Laboratory of Functional Nanomaterials NanoFun nr POIG.02.02.00-00-025/09 (Innovative Economy Operational Programme, Priority
Axis 2: R\&D Infrastructure, Action 2.2: Support of Formation of Common Research Infrastructure of Scientific Units).

This work was supported by the National Science Centre based on decision number DEC-2013/11/B/ NZ9/01761, as well as by the European Regional Development Fund through the Innovative Economy for Poland 2007-2013 (WND-POIG.01.03.01-00-101/08 POLAPGEN-BD "Biotechnological tools for breeding cereals with increased resistance to drought"), and the KNOW RNA Research Centre in Poznan (01/ KNOW2/2014).

\section{LITERATURE}

Alaba S, Piszczalka P, Pietrykowska H, Pacak AM, Sierocka I, Nuc PW, Singh K, Plewka P, Sulkowska A, Jarmolowski A, Karlowski WM, Szweykowska-Kulinska Z (2015) The liverwort Pellia endiviifolia shares microtranscriptomic traits that are common to green algae and land plants. New Phytologist 206: 352-367. doi: 10.1111/ nph. 13220

Allen RS, Li J, Alonso-Peral MM, White RG, Gubler F, Millar AA (2010) MicroR159 regulation of most conserved targets in Arabidopsis has negligible phenotypic effects. Silence 1: 18-18. doi: 10.1186/1758-907X-1-18

Barciszewska-Pacak M, Milanowska K, Knop, K, Bielewicz D, Nuc P, Plewka P, Pacak AM, Vazquez F, Karlowski W, Jarmolowski A, Szweykowska-Kulinska Z (2015) Arabidopsis microRNA expression regulation in a wide range of abiotic stress responses. Front Plant Sci 6: 410. doi: 10.3389/fpls.2015.00410.

Baumberger N, Baulcombe D (2005) Arabidopsis ARGONAUTE1 is an RNA Slicer that selectively recruits microRNAs and short interfering RNAs. Proc Natl Acad Sci USA 102: 11928-11933. www.pnas. org/cgi/doi10.1073/pnas.0505461102

Carbonell A, Fahlgren N, Garcia-Ruiz H, Gilbert KB, Montgomery TA, Nguyen T, Cuperus JT, Carrington JC (2012) Functional analysis of three Arabidopsis ARGONAUTES using slicer-defective mutants. Plant Cell 24: 3613-3629. doi: 10.1105/tpc.112.099945

Dai X, Zhao PX (2011) psRNATarget: a plant small RNA target analysis server. Nucleic Acids Res 39: W155-W159. doi: 10.1093/nar/ gkr319.

Deng, W, Nickle DC, Learn GH, Maust B, Mullins JI (2007) ViroBLAST: a stand-alone BLAST web server for flexible queries of multiple databases and user's datasets. Bioinformatics 23: 2334-2336. doi: 10.1093/bioinformatics/btm331

Devaux P, Adamski P, Surma M (1992) Inheritance of seed set in crosses of spring barley and Hordeum bulbosum L. Crop Science 32: 269-271.

Endo Y, Iwakawa Ho, Tomari Y (2013) Arabidopsis ARGONAUTE7 selects miR390 through multiple checkpoints during RISC assembly. EMBO Reports 14: 652-658. doi: 10.1038/embor.2013.73

Fahlgren N, Howell M.D, Kasschau KD, Chapman EJ, Sullivan CM, Cumbie JS, Givan SA, Law TF, Grant SR, Dangl JL, Carrington JC (2007) High-throughput sequencing of Arabidopsis microRNAs: evidence for frequent birth and death of MIRNA genes. PLoS One 2: e219. doi: 10.1371/journal.pone.0000219.

Fahlgren N, Montgomery TA, Howell MD, Allen E, Dvorak SK, Alexander AL, Carrington IC (2006) Regulation of AUXIN RESPONSE FACTOR3 by TAS3 ta-siRNA affects developmental timing and patterning in Arabidopsis. Curr Biol 16: 939-944. doi: 10.1016/j. cub.2006.03.065

Fan T, Li X, Yang W, Xia K, Ouyang J, Zhang M (2015) Rice osamiR171c Mediates Phase Change from Vegetative to Reproductive Development and Shoot Apical Meristem Maintenance by Repressing Four OsHAM Transcription Factors. PLoS One 10: e0125833. doi: 10.1371 /journal.pone.0125833.

Garcia-Ruiz H, Carbonell A, Hoyer JS, Fahlgren N, Gilbert KB, Takeda A, Giampetruzzi A, Garcia Ruiz MT, McGinn MG, Lowery N, Martinez Baladejo MT, Carrington JC (2015) Roles and programming of Arabidopsis ARGONAUTE proteins during Turnip mosaic virus infection. PLOS Pathog 11: e1004755. doi: 10.1371/journal. ppat.1004755.

German MA, Luo S, Schroth G, Meyers BC, Green PJ (2009) Construction of Parallel Analysis of RNA Ends (PARE) libraries for the study of cleaved miRNA targets and the RNA degradome. Nature Protocols 4: 356-362. doi: 10.1038/nprot.2009.8

Hackenberg M, Shi B-J, Gustafson P, Langridge P (2013) Characterization of phosphorus-regulated miR399 and miR827 and their isomirs in barley under phosphorus-sufficient and phosphorus-deficient conditions. BMC Plant Biol 13: 214. doi: 10.1186/1471-2229-13-214

Iki T, Yoshikawa M, Nishikiori M, Jaudal MC, Matsumoto-Yokoyama E, Mitsuhara I, Meshi T, Ishikawa M (2010) In vitro assembly of 
plant RNA-induced silencing complexes facilitated by molecular chaperone HSP90. Mol Cell 39: 282-291. doi: 10.1016/j.molcel.2010.05.014.

Kozomara A, Griffiths-Jones S. (2011) miRBase: integrating microRNA annotation and deep-sequencing data. Nucleic Acids Res 39 (Database issue): D152-D157. doi: 10.1093/nar/gkq1027.

Kruszka K, Pacak A, Swida-Barteczka A, Stefaniak K, Kaja E, Sierocka I, Karlowski W, Jarmolowski A, Szweykowska-Kulinska Z (2013) Developmentally regulated expression and complex processing of barley pri-microRNAs. BMC Genomics 14: 34. doi: 10.1186/14712164-14-34.

Kruszka K, Pacak A, Swida-Barteczka A, Nuc P, Alaba S, Wroblewska Z, Karlowski W, Jarmolowski A, Szweykowska-Kulinska Z (2014) Transcriptionally and post-transcriptionally regulated microRNAs in heat stress response in barley. J Exp Bot 65: 6123-6135. doi: $10.1093 / \mathrm{jxb} / \mathrm{eru} 353$

Lin SI, Santi C, Jobet E, Lacut E, El Kholti N, Karlowski WM, Verdeil JL, Breitler JC, Perin C, Ko SS, Guiderdoni E, Chiou TJ, Echeverria M (2010) Complex regulation of two target genes encoding SPX-MFS proteins by rice miR827 in response to phosphate starvation. Plant Cell Physiol 51: 2119-2131. doi: 10.1093/pcp/pcq170.

Liu N, Wu S, Van Houten J, Wang Y, Ding B, Fei Z, Clarke TH, Reed JW, van der Knaap E (2014) Down-regulation of AUXIN RESPONSE FACTORS 6 and 8 by microRNA 167 leads to floral development defects and female sterility in tomato. $J$ Exp Bot 65: 2507-2520. doi: 10.1093/jxb/eru141.

Lukasik A, Pietrykowska H, Paczek L, Szweykowska-Kulinska Z, Zielenkiewicz P (2013) High-throughput sequencing identification of novel and conserved miRNAs in the Brassica oleracea leaves. BMC Genomics 14: 801. doi: 10.1186/1471-2164-14-801.

Mallory A, Vaucheret H (2010) Form, function, and regulation of ARGONAUTE proteins. Plant Cell 22: 3879-3889. doi: 10.1105/ tpc.110.080671.

Marin E, Jouannet V, Herz A, Lokers, AS, Weijers D, Vaucheret H, Nussaume L, Crespi MD, Maizel A (2010) miR390, Arabidopsis TAS3 tasiRNAs, and their AUXIN RESPONSE FACTOR targets define an autoregulatory network quantitatively regulating lateral root growth. The Plant Cell 22: 1104-1117. http://dx.doi.org/10. 1105 tpc. 109.072553

Pandey R, Joshi G, Bhardwaj AR, Agarwal M, Katiyar-Agarwal S (2014) A comprehensive genome-wide study on tissue-specific and abiotic stress-specific miRNAs in Triticum aestivum. PLoS One 9: e95800. doi: 10.1371/journal.pone.0095800.
Schirle NT, MacRae IJ (2012) The crystal structure of human Argonaute2. Science 336: 1037-1040. doi: 10.1126/science.1221551.

Schreiber AW, Shi B-J, Huang C-Y, Langridge P, Baumann U (2011) Discovery of barley miRNAs through deep sequencing of short reads. BMC Genomics 12: 1.

Si-Ammour A, Windels D, Arn-Bouldoires E, Kutter C, Ailhas J, Meins F, Jr, Vazquez F (2011) miR393 and secondary siRNAs regulate expression of the TIR1/AFB2 auxin receptor clade and auxin-related development of Arabidopsis leaves. Plant Physiol 157: 683-691. doi: 10.1104/pp.111.180083.

Sobkowiak L, Karlowski W, Jarmolowski A, Szweykowska-Kulinska Z (2012) Non-Canonical Processing of Arabidopsis pri-miR319a/b/c Generates Additional microRNAs to Target One RAP2.12 mRNA Isoform. Front Plant Sci 3: 46. doi: 10.3389/fpls.2012.00046.

Spanudakis E, Jackson S (2014) The role of microRNAs in the control of flowering time. J Exp Bot 65: 365-380. doi: 10.1093/jxb/ert453.

Wang L, Gu X, Xu D, Wang W, Wang H, Zeng M, Chang Z, Huang H, Cui X (2011) miR396-targeted AtGRF transcription factors are required for coordination of cell division and differentiation during leaf development in Arabidopsis. J Exp Bot 62: 761-773. doi: $10.1093 /$ jxb/erq307.

Xie K, Shen J, Hou X, Yao J, Li X, Xiao J, Xiong L (2012) Gradual increase of miR156 regulates temporal expression changes of numerous genes during leaf development in rice. Plant Physiol 158: 1382-1394. doi: 10.1104/pp.111.190488.

Xie K, Wu C, Xiong L (2006) Genomic organization, differential expression, and interaction of SQUAMOSA promoter-binding-like transcription factors and microRNA156 in rice. Plant Physiol 142: 280-293. doi: 10.1104/pp.106.084475.

Zadoks JC, Chang TT, Konzak CF (1974) A decimal code for the growth stages of cereals. Weed Res 14: 415-421.

Zhang X, Niu D, Carbonell A, Wang A, Lee A, Tun V, Wang Z, Carrington JC, Chang CE, Jin H (2014) ARGONAUTE PIWI domain and microRNA duplex structure regulate small RNA sorting in Arabidopsis. Nat Commun 5: 5468. doi: 10.1038/ncomms6468.

Zielezinski A, Dolata J, Alaba S, Kruszka K, Pacak A, Swida-Barteczka A, Knop K, Stepien A, Bielewicz D, Pietrykowska H, Sierocka I, Sobkowiak L, Lakomiak A, Jarmolowski A, Szweykowska-Kulinska Z, Karlowski WM (2015) mirEX 2.0-an integrated environment for expression profiling of plant microRNAs. BMC Plant Biol 15: 144. doi: 10.1186/s12870-015-0533-2 